\title{
Foggy Images Classification Based On Features Extraction and SVM
}

\author{
Yuanyuan Zhang, Guangmin Sun, Qian Ren and Dequn Zhao \\ Department of Electronic Engineering \\ Beijing University of Technology \\ Beijing, 100124, China \\ e-mail: gmsun@bjut.edu.cn
}

\begin{abstract}
An algorithm of foggy image classification is presented in this paper. First, the RGB images are converted to HSI images and next we analysis the distribution of the histograms of $\mathrm{H}, \mathrm{S}$, I plane separately, from which we extract the variance of each plane under different foggy conditions as the HSI model features. Second, the dichromatic atmospheric scattering model is introduced and based on this model we develop an algorithm for computing the angular deviation of different foggy images compared to clear day image as another feature. Finally, we use this feature set to train a multi-class SVM classifier to classify four different levels of foggy images. Experiment results show that the algorithm is more than $\mathbf{9 0 \%}$ accurate.
\end{abstract}

Keywords-HSI model; histogram; dichromatic atmospheric scattering model; SVM

\section{INTRODUCTION}

Most computer vision systems are designed to work well in clear weather conditions and it is assumed that the observer is surrounded by invariant atmospheric medium. However, in many cases we have to deal with bad weather situations such as haze and fog. In this paper we present a method to solve the problem of classifying four levels of foggy images of equipment of power transmission cables, in which the conditions have been divided into four classes of clear, light foggy, medium foggy and heavy foggy. This method consists of two main steps of image processing of features extraction and pattern recognition of training a multi-class classifier.

In the first step of features extraction we analysis the histogram of the images in HSI [1] color model. Useful applications of histogram include contrast enhancement of histogram equalization [2], histogram modification of medical image processing [3] and speech recognition [4], in which histogram has been widely used due to its simplicity and effectiveness. In this paper we will analysis the distribution of histogram and we find that the variance of each plane of HSI color model implies the different contrast gradient of the images under different foggy conditions so that the variance can be used as the ideal feature of HSI color model. As for another feature is based on the dichromatic atmospheric scattering model [5] and we compute the angular deviation between foggy image and clear one as the feature. The second step is pattern recognition and we choose SVM [6] as the training model. After step 1 we can get the features as the training vector and by processing hundreds of different foggy images we can get the training set to train a multi-class SVM classifier. Finally, we can use this method to process any unknown image through the same procedure and get its correct foggy level with the accuracy of $95 \%$.

This paper can be divided into four sections. Section 1 is the introduction of the backgrounds and applications of the main technology and the overlook of the procedure of this method. In section 2 the first main step of features extraction will be discussed and the experimental result will be shown as well. Section 3 is the second main step of training a fourclass SVM classifier and we can see clearly the accuracy of this method of the experimental result is at least $95 \%$. In the last section 4 we will conclude the advantages and disadvantages of this method and give the possible future development of it.

\section{FEATURES EXTRACTION}

In this section we firstly introduce the concept of HSI color model and how to transform RGB image into HSI image, then draw the histogram of each $\mathrm{H}, \mathrm{S}$, I plane and extract the variance of the histogram as the feature and explain the meaning of it as in the foggy weather conditions. All that mentioned above will be included in section $\mathrm{A}$. In section $\mathrm{B}$ we will introduce the theory of the dichromatic atmospheric scattering model and how we get the angular deviation as another feature.

\section{A. Features of HSI Color Model}

There are two categories of common used color model: the hardware oriented color model and the color processing application oriented color model [7]. The RGB color model is widely used due to its hardware orientation so that the image can be easily stored and displayed in computers. But it is non-linear and inconvenient in visual perception. Therefore another approach of color model using HSI has been considered to solve this problem.

HSI color model matches nicely with the human visual perception and is suitable for describing and interpreting the image. $\mathrm{H}, \mathrm{S}$ and I represent hue, saturation, and intensity respectively. Hue is a color attribute which describes a pure color (pure red, yellow or green); saturation is a measure of the degree to which a pure color is diluted by white light; intensity describes color sensation which is influenced by light. The separation of the light component from color information is proved to have advantages in applications such as image processing. In order to transform RGB color model into HSI color model, we need to get the values of red, green and blue directly from each pixel of the original image and use the (1) (2) [8]. 


$$
\left\{\begin{array}{l}
I=\frac{1}{3}(R+G+B) \\
S=1-\frac{3}{(R+G+B)}[\min (R, G, B)] \\
H= \begin{cases}\theta & B \leq G \\
2 \pi-\theta & B>G\end{cases}
\end{array}\right.
$$$$
\theta=\arccos \left\{\frac{\frac{1}{2}[(R-G)+(R-B)]}{\left[(R-G)^{2}+(R-B)(G-B)\right]^{1 / 2}}\right\}
$$

After we get the HSI color model the next step is to draw the histogram of each $\mathrm{H}, \mathrm{S}$ and I plane. The histogram is a statistical report map and represented by a series of varying height longitudinal stripes or segment of data distribution. Usually, the abscissa indicates the data type, and the vertical axis represents the distribution. In the grayscale images, the abscissa of histogram is the distribution of the grayscale, the vertical axis represents the quantity of the pixel under each grayscale. Histogram is a very effective tool on describing the features of the color image. It describes the statistical distribution of the color features of the image, and due to its invariance of translation, scaling and rotation it is widely used in many fields.

In this paper we use the histogram to describe the mathematical properties of the variance of the foggy image under HSI color model. This feature represents the contrast of each H, S, I plane under different level foggy conditions. Hue is the pure color of the image and in clear weather its distribution of the histogram is uniform and smooth while in foggy weather it gets narrower and shaper as the foggy level gets higher. The situation of the saturation and intensity is the same due to the foggy condition add more bright white light into the image and the image gets whiter as the foggy level gets higher and the feature of the variance gets smaller. The illustration of this situation can be seen in figure 1 .

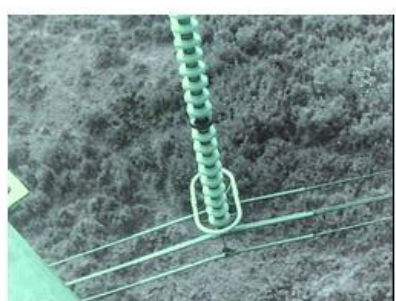

(a) clear

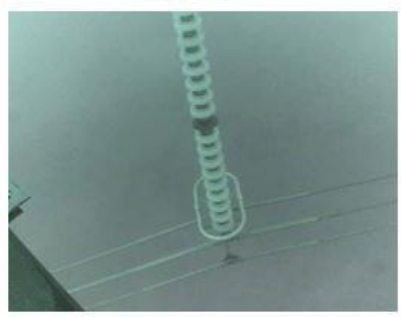

(c) medium

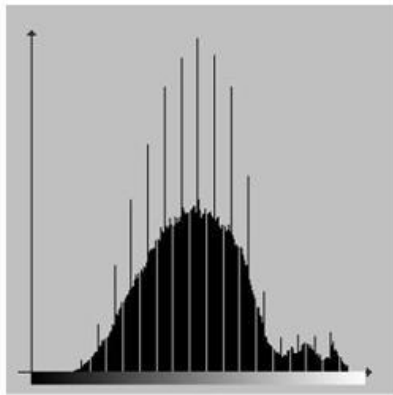

(a) clear

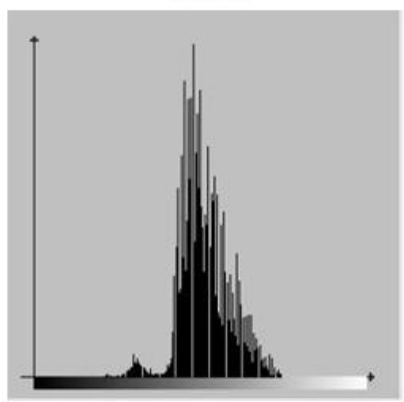

(c) medium

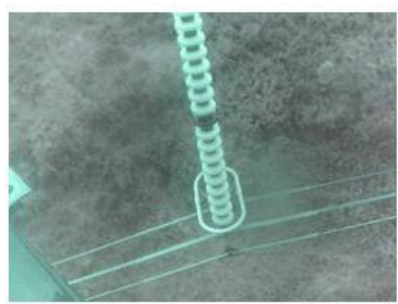

(b) light

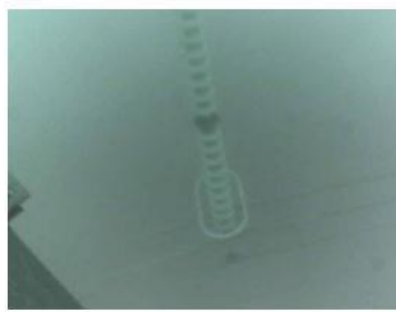

(d) heavy

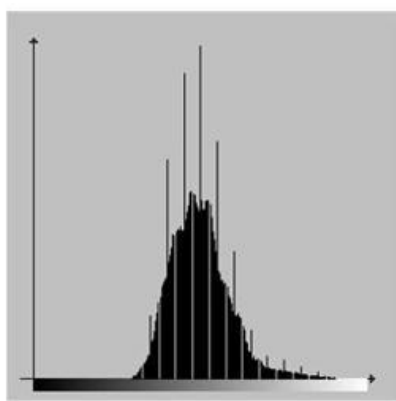

(b) light

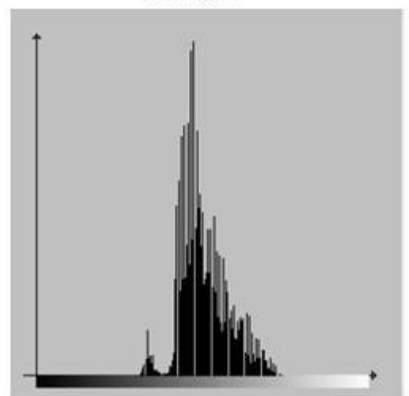

(d) heavy
Figure 1. The illustration of the contrast under different foggy level.

\section{B. Features Of Dichromatic Atmospheric Scattering Model}

On the basis of the dichromatic atmospheric scattering model, a method was proposed to the identification of weather especially for obstructions to vision phenomena. The method only requires the same scene with sunny and vision blocked weather phenomena of dichromatic atmospheric scattering model digital image. In this section we will introduce the basic theory of dichromatic atmospheric scattering model and propose the method to calculate pixel vector angle between the pixel and luminance information in the selected area as the feature in this model.

The intensity an object received from the light reaches the camera to get the image can be divided into two parts. 
One of the intensity is the directly transmitted light component $D(d, \lambda)$, which is the scattering attenuation of atmospheric aerosol particles through the optical visual range to reach the photosensitive element of the process of the camera under foggy weather [9]. The intensity of the directly transmitted light component described as $p=\frac{E_{\infty} \gamma e^{-\beta d}}{d^{2}}$

The air light and ground surface reflected light through scattering and refraction effects of atmospheric aerosol particles and air molecules also has transmitted part of the light into the camera and this is called the atmospheric light scattering component $A(d, \lambda)$, which can be described as $q=E_{\infty}\left(1-e^{-\beta d}\right)$ (4). The dichromatic model can then be written as $\mathbf{E}=p \hat{\mathbf{D}}+q \hat{\mathbf{A}}$ (5) and we can see clearly in figure 2 .

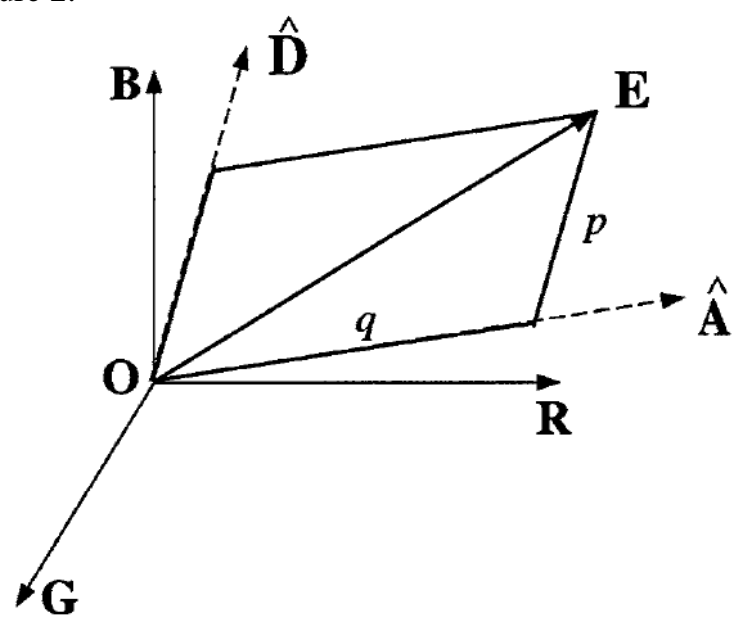

Figure 2. Dichromatic atmospheric scattering model.

The feature of the dichromatic atmospheric scattering model is the angular deviation of the vector angle of the same pixel between two different foggy levels in the RGB color model. The angular deviation of the vector angle varies a lot in different foggy conditions and can be seen clearly in figure 3 .

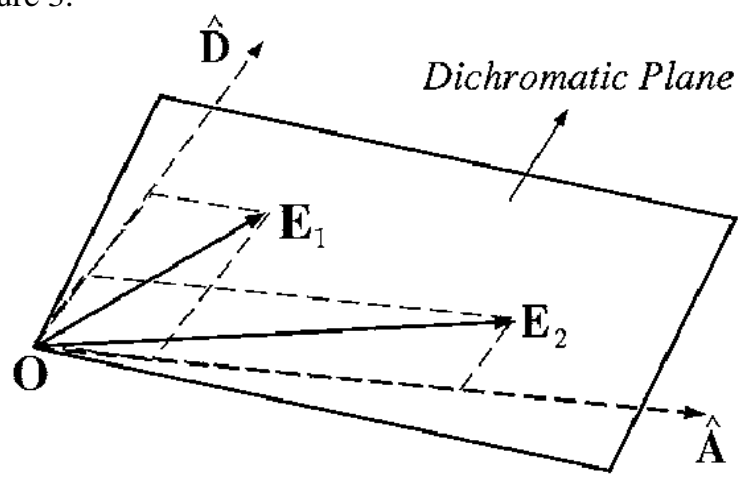

Figure 3. The angular deviation of the vector angle.
The vector coordinate position of the same pixel $\mathbf{E}$ in two different foggy levels in RGB color model is illustrated as above. The different vector $\mathbf{E}_{1}$ and $\mathbf{E}_{2}$ is the intensity of the pixel in two different foggy levels. As we can see in the figure the vector $\mathbf{E}$ in the dichromatic plane can be decomposed in two axes of is the directly transmitted light coordinate direction $\hat{\mathbf{D}}$ and the atmospheric light scattering coordinate direction $\hat{\mathbf{A}}$. In the theory of the dichromatic atmospheric scattering model we know that the directly transmitted light component $D(d, \lambda)$ of the same pixel is determined only by the transmission distance and has nothing to do with the weather conditions, so its value remains the same in different foggy level. Meanwhile the atmospheric light scattering component $A(d, \lambda)$ changes with the different foggy conditions, because the scattering and refraction effects of the air light and ground surface reflection get bigger as the fog gets severer. From this we can draw that the foggy level of the pixel $\mathbf{E}_{2}$ is severer than $\mathbf{E}_{1}$ and we can calculate the angular deviation of them as our feature.

\section{SvM ClassifiCATION}

Section III is the discussion of the multi-class SVM classifier, which is the second main step, and also be divided into two parts. An overview of Support Vector Machine (SVM) is given in section A. The use of LIBSVM is mainly discussed in section $\mathrm{B}$, which is the software we use to build the model of a four-class SVM classifier, and gives the results of the classification.

\section{A. Overview of SVM}

SVM was a new general-purpose machine learning method based on statistical learning theory, and it was built under the theory framework and general approach of machine learning with limited samples. Its basic thought was to transform the input space to a high-dimension one by using the non-linear transformation defined by inner product function and to find a non-linear relationship between the input variables and the output ones in high-dimension [10].

SVM uses a linear model to implement nonlinear class boundaries through some nonlinear mapping input vectors into a high-dimensional feature space. The linear model constructed in the new space can represent a nonlinear decision boundary in the original space. In the new space, an optimal separating hyper plane is constructed. Thus, SVM is known as the algorithm to find a special kind of a linear model, the maximum margin hyper plane. The maximum margin hyper plane gives the maximum separation between decision classes. The training examples that are close to the maximum margin hyper plane are called support vectors. All other training examples are irrelevant for defining the binary class boundaries [11].

\section{B. Use of LIBSVM and Results}

LIBSVM is a fast and efficient pattern recognition and regression software package developed by professor Chih- 
Jen Lin of Taiwan University. It is easy and simple for beginners to use without knowing the underlying theory behind SVM. Lin not only provides a compiled executable file in Windows system which can be used in DOS, but also offers the source codes so that developers can improve and modify them for their projects under different development environment and operating system. We can easily solve the problems of classification by using C-SVM and V -SVM and regression by using $\varepsilon-S V R$ and $v-S V R$ of LIBSVM. In the application of SVM we need to adjust parameters many times in order to get a trained classifier, LIBSVM provides the function of Cross Validation which can get the best parameters under the optimal classification accuracy.

The recommended procedure can be seen as follows.

Step 1 Transform data to the format of an SVM package.

The data here are the features we get from section II. Each feature is an attribute of a vector and the sample set consists of a number of vectors of two different classes. As for Hong He, we extract four features to make up vectors of sample set. The format of an SVM package is like this:

[label] [index1]:[value1] [index2]:[value2] ...

Label is the class of the vector. When it is two-class classification, label is $+1 /-1$ or $+1 / 0$, when it's multi-class cases, label is the integer of $1 \ldots n$. Index is the index of features and must be in ascending order. Value is the features.

Step 2 Conduct simple scaling on the data.

Scaling before applying SVM is very important. The main advantage of scaling is to avoid attributes in greater numeric ranges dominating those in smaller numeric ranges. Another advantage is to avoid numerical difficulties during the calculation. Because kernel values usually depend on the inner products of feature vectors, e.g. the linear kernel and the polynomial kernel, large attribute values might cause numerical problems. We recommend linearly scaling each attribute to the range $[-1 ;+1]$ or $[0 ; 1]$.

Step 3 Consider the RBF kernel

In general, the RBF kernel is a reasonable first choice.

Step 4 Use cross-validation to find the best parameter $C$ and $\gamma$.

There are two parameters for an RBF kernel: $C$ and $\gamma$. The goal is to identify good $C$ and $\gamma$ so that the classifier can accurately predict unknown data (i.e. Testing data). Note that it may not be useful to achieve high training accuracy (i.e. a classifier which accurately predicts training data whose class labels are indeed known). As discussed above, a common strategy is to separate the data set into two parts, of which one is considered unknown. The prediction accuracy obtained from the "unknown" set more precisely reflects the performance on classifying an independent data set. An improved version of this procedure is known as crossvalidation.

Step 5 Use the best parameter $C$ and $\gamma^{\gamma}$ to train the whole training set and get the model text which contains the corresponding parameters and support vectors.

Step 6 Use the model text to do the classification.
The whole procedure can be done in DOS by the tool easy.py of LIBSVM and it's shown in figure 4.

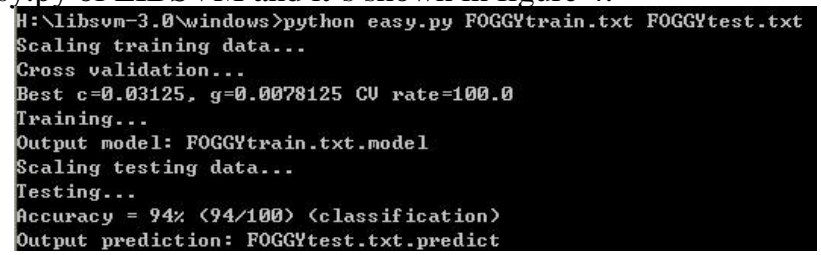

Figure 4. The result of foggy images using LIBSVM.

\section{CONCLUSION AND SUMMARY}

The conclusion and summary of the paper is given in Section IV. The method of features extraction has provided us with the color model and dichromatic atmospheric scattering model, which can analysis the foggy images in details and indicate the different features under different foggy level. The use of SVM can solve the problem of the limited small training sample sets and get a rather ideal accuracy of predict result.

However, the method is limited in the use of foggy weather and the features aren't good enough to describe the whole information of images under foggy weather. In the future we will continue to extract more other features to complete this method.

\section{REFERENCES}

[1] Castleman K R. Digital image processing. Prentice-Hall, 1996.

[2] T.-K. Kim, J.-K. Paik, and B.-S. Kang, "Contrast enhancement system using spatially adaptive histogram equalization with temporal filtering," IEEE Trans. on Consumer Electronics, vol. 44, no. 1, pp. 82-86, Feb. 1998.

[3] S. M. Pizer, "The medical image display and analysis group at the university of north carolina: Reminiscences and philosophy," IEEE Trans. Med. Imag., vol. 22, pp. 2-10, Jan. 2003.

[4] A. de la Torre, A. M. Peinado, J. C. Segura, J. L. Perez-Cordoba, M. C. Benitez, and A. J. Rubio, "Histogram equalization of speech representation for robust speech recognition," IEEE Trans. Speech Audio Processing, vol. 13, pp. 355-366, May 2005.

[5] Srinivasa G. Narasimhan, Shree K. Nayar. Chromatic Framework for Vision in Bad Weather [J].IEEE Conference on Computer Vision and Pattern Recognition, 2000, 598-605.

[6] Hongjun Zheng, Xu Zhou and Duyan Bi, "Introduction statistical learning theory and support vector machines", Modern Electronics Technique, No. 4, pp.59-61, 2003.

[7] R.C.Gonzalez and R.E.Woods, "Digital Image Processing", AddisonWesley Publishing company, Inc., 1993.

[8] R. C. Gonzalez, and R. E. Woods, Digital Image Processing, Second Edition. Pearson Education, 2nd Ed., New Jersey, 2002.

[9] Srinivasa G. Narasimhan, Shree K. Nayar. Chromatic Framework for Vision in Bad Weather [J].IEEE Conference on Computer Vision and Pattern Recognition, 2000, 598-605.

[10] Y. C. Lee, "Application of support vector machines to corporate credit rating prediction," Expert Systems with Applications, Elsevier Science, vol.33, pp.:67-74, 2007.

[11] C.J.C. Burges, "A tutorial on support vector machines for pattern recognition," Data Mining and Knowledge Discovery, Springer Netherlands, vol. 2, pp.:955 974, 1998. 